Arthroskopie $2010 \cdot 23: 5-5$

DOI 10.1007/s00142-009-0538-7

Online publiziert: 20. Dezember 2009

(c) Springer-Verlag 2009

\author{
H. Mayr \\ Orthopädische Chirurgie München (OCM), München
}

\title{
Vorderes und hinteres Kreuzband
}

Liebe Kolleginnen und Kollegen,

in Deutschland, Österreich und der Schweiz dürften jährlich zwischen 43.000 und 55.00o Primärrekonstruktionen und Revisionsoperationen der Kreuzbänder vorgenommen werden. Die ursächliche Verletzung betrifft überwiegend jüngere Menschen und hat neben dem persönlichen Schicksal auch volkswirtschaftliche Relevanz.

Die Kniebandchirurgie ist einem permanenten Wandel unterworfen. Zum einen nimmt die Präzision bzgl. Diagnostik und Indikationsstellung zu. Zum anderen werden die Operationstechniken weniger invasiv und differenzierter.

Die erste Beschreibung der Anatomie und Funktion der Kreuzbänder des Kniegelenks und die Bezeichnung „ligamenta genu cruciata“ entstammen einem Werk des Claudius Galen von Pergamon (199-129 v. Chr.). Die erste biomechanische Arbeit über das Kniegelenk mit Untersuchung der stabilisierenden Funktion der Kreuzbänder in sagittaler Richtung wurde von den Gebrüdern Weber im Jahr 1836 veröffentlicht. 1885 wurde erstmals eine chirurgische Intervention an den Kreuzbändern schriftlich dokumentiert. Die Komplexität einer Knieinstabilität wurde 1938 von Palmer ganzheitlich beschrieben. In seiner Veröffentlichung „On the injuries of the ligaments of the knee joint" beschreibt er Anatomie, Biomechanik und Behandlung ligamentärer Knieverletzungen. Campbell publizierte 1939 erstmals die Verwendung der Patellasehne als intraartikuläre Plastik für ein frisch rupturiertes vorderes Kreuzband. Macey beschreibt im gleichen Jahr die Verwendung der Hamstringsehnen (Semitendinosus- und Grazilissehne) für den Kreuzbandersatz.

Viele Studien zeigen, dass ein stabiles Knie für den langfristigen Erhalt des Gelenks erforderlich ist.

Die Invasivität des Eingriffs wurde in den 8oer Jahren des letzten Jahrhunderts erheblich reduziert. In den letzten beiden Jahrzehnten orientieren sich die Kniebandrekonstruktionen zunehmend an der funktionellen Anatomie des Gelenks. Besonders bei hinteren Knieinstabilitäten wird in jüngerer Zeit die Komplexität der Verletzung differenzierter diagnostiziert, bewertet und therapiert. Die Revisionschirurgie des vorderen und hinteren Kreuzbandes unterliegt heute einem diagnostischen und therapeutischen Algorithmus.

\section{- Die anatomische Rekonstruktion der Kreuzbänder ist ein wesentliches Ziel.}

In diesem Zusammenhang hat die Doppelbündeltechnik an Bedeutung gewonnen. Bei Komplexverletzungen wird die
Wiederherstellung der extraartikulären Stabilisatoren angestrebt. Zur objektiven Beurteilung der Laxität und der Operationsergebnisse werden derzeit Messgeräte entwickelt, die Rotationsinstabilitäten messen können.

Die operative Behandlung der vorderen und hinteren Kreuzbandruptur sowie die Sanierung von Komplexinstabilitäten sind heute eine Domäne der arthroskopischen und minimalinvasiven Therapie.

Wir hoffen, Ihnen einen aktuellen Überblick über diese Zusammenhänge und Techniken zu geben,

Ihr

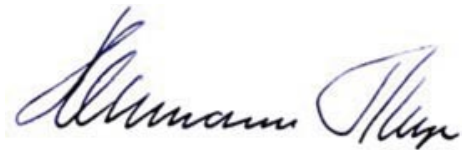

Hermann Mayr

\section{Korrespondenzadresse \\ PD Dr. H. Mayr}

Orthopädische Chirurgie München (OCM)

Steinerstr. 6, 81369 München

team.mayr@ocm-muenchen.de 